\title{
A Novel Face Hallucination with an Error Regression Model and MPCA in RGB Color Space
}

\author{
Krissada Asavaskulkiet and Phumin Kirawanich
}

\begin{abstract}
This paper proposes a novel face super-resolution reconstruction (hallucination) technique, color face images reconstruction of RGB space with an error regression model in multi-linear principal component analysis (MPCA). From hallucination framework, many color face images are explained in RGB space. Then, they can be naturally described as tensors or multi-linear arrays. In this way, the error regression analysis is used to find the error estimation which can be obtained from the existing $L R$ in tensor space. The framework consists of learning and hallucinating process. In learning process is from the mistakes in reconstruct face images of the training dataset by MPCA, then finding the relationship between input and error by regression analysis. In hallucinating process uses normal method by back-projection of MPCA, after that the result is corrected with the error estimation. In this contribution we show that our hallucination technique can be suitable for color face images both in RGB space. By using the MPCA subspace with error regression model, we can generate photorealistic color face images. Our approach is demonstrated by extensive experiments with high-quality hallucinated color faces. In addition, our experiments on face images from FERET database validate our algorithm
\end{abstract}

Index Terms-MPCA, error regression model

\section{INTRODUCTION}

Face super-resolution (SR), also known as face hallucination, aims to recover high quality, high resolution images of human faces from low-resolution, blurred, and degraded images or video. It is used to render a high-resolution (HR) face image from multiple low-resolution (LR)[1] and the color face image can be called tensor objects, 3-D (third-order tensor) with column, row, and color modes [2]. In addition, the most active area of biometrics research, namely, that of face recognition, 3-D face detection and recognition using 3-D information with column, row, and depth modes, in other words, a third-order tensor, has emerged as an important research direction $[3,4$, 5].

The principal component analysis (PCA) is proposed to fit the input face image as a linear combination of the training LR face images, and then the HR image is synthesized by replacing the training LR face images with the HR counterparts while retaining the same combination weights[6], [7]. Gunturk et al.[8] apply PCA to determine the prior model. With learning-based method for face hallucination, a kernel PCA is applied for deriving prior

Manuscript received September 25, 2012; revised October 25, 2012.

The authors are with the Department of Electrical Engineering, Mahidol University, Salaya, Nakhon Pathom, 73170 Thailand (e-mail: bankachieve@gmail.com) knowledge about the face class [9].

In [10], they developed a two-step statistical modeling approach that integrates both a global parametric model and local nonparametric model. The global constraint assumes a Gaussian distribution learnt by PCA. The local constraint utilizes a patch-based nonparametric Markov network to learn the statistical relationship between the global face image and the local features. PCA was applied on both HR and LR, and the global image, which was similar to the original HR image, was reconstructed under a maximum a posteriori (MAP) criterion. Second, a linear model between the residual image and the LR residual image are built, and following a Markov Random Field (MRF) prior, the optimal residua image was estimated under a MAP criterion again.

The MPCA is a general extension of traditional linear methods such as PCA or matrix SVD [11]. There is a strong analogy between several properties of the matrix and the higher-order tensor decomposition and multi-linear generalization of the symmetric eigenvalue decomposition for pair-wise symmetric tensors.

This paper proposes an alternative framework, which consists two-phases, there is learning and hallucinating phase. An important issue is that we take advantage of MPCA and error regression model. The error regression model is added in training process for improving face image reconstruction. In the first phase, learning process is learning the error of the training dataset in face image reconstruction by MPCA. Considering the error of reconstruction, the system does not only learn the correct information but it also learn to correct the wrong information then builds error regression model for learning. Furthermore, color face images are reconstructed from corrected data while error data are corrected by recognizing from the error regression model. In this model, this is finding the relationship between input and error by regression analysis.

The rest of this paper is organized as follows. In Section II, we explain the basic notation and introduce the basic idea in multi-linear principal component analysis (MPCA) in Section III. In Section IV, we propose a novel color hallucination method, using the error regression model in MPCA for RGB color space. Some experimental results are presented in Section V. Finally, conclusions are given in Section VI

\section{BASIC NOTATION}

In this paper, scalars are denoted by lower case letters $(a, b, \ldots)$, vectors by italic upper-case case letters $(A, B, \ldots)$, matrices by bold upper-case letters $(A, B, \ldots)$, and higher-order tensors by calligraphic upper-case letters 
$(\mathcal{A}, \mathcal{B})$. denotes the transpose of a matrix denotes the pseudo-inverse.

\section{BASIC IDEA IN MULTI-LineAR PRINCIPAL COMPONENT ANALYSIS (MPCA)}

The basic idea in MPCA solution to the problem of dimensionality reduction for tensor objects is introduced [11]. In Fig. 1, we show the basic idea in MPCA, an n-mode unfolding of a tensor. An $N$ th-order tensor is denoted as $\mathcal{A}$ $\in \mathrm{R}^{I_{1} \times I_{2} \times \ldots I_{N}}$ for $I_{n}=1, \ldots, N$. The $n$-mode vectors of $\mathcal{A}$ are defined as the $I_{n}$-dimensional vectors obtained from $\mathcal{A}$ by varying the index $i_{n}$ while keeping all the other indices fixed. The unfolding of $\mathcal{A}$ along the $n$-mode is denoted as $\mathrm{A}_{(\mathrm{n})} \in \mathrm{R}^{\ln \times\left(I_{1} \times I_{2} \times \ldots . . I_{N}\right)}$ and the column vectors of $\mathrm{A}_{(\mathrm{n})}$ are the $n$-mode vectors of $\mathcal{A}$.

Let the set of tensors be $\left\{\mathcal{A}_{m}, m=1, \ldots, M\right\}$ and the total scatter of these tensors is defined as

$$
\psi_{\mathrm{A}}=\sum_{m=1}^{M}\left\|A_{m}-\bar{A}\right\|^{2}
$$

where $\overline{\mathcal{A}}$ is the mean tensor calculated as

$$
\overline{\mathcal{A}} \quad=(1 / M) \sum_{m=1}^{M} \mathcal{A}_{\mathrm{m}}
$$

Then, the total scatter matrix of these samples can be defined as

$$
C_{A}=\sum_{m=1}^{M}\left(A_{m(n)}-\bar{A}_{(n)}\right)\left(A_{m(n)}-\bar{A}_{(n)}\right)^{T},
$$

where $\mathrm{A} m(n)$ is the $n$-mode unfolded matrix of $\mathrm{A} m$.

The main objective of MPCA is to define a multi-linear transformation $\tilde{\mathrm{U}}^{(\mathrm{n})}$ which denote $I_{n} \times P_{n}$ matrix containing the orthornormal $n$-mode basis vectors and the matrix $\tilde{\mathrm{U}}^{(\mathrm{n})}$ is $n$th projection matrix, $n=1, \ldots, N$. It can map the original tensor space $\mathrm{R}^{I_{1}} \otimes \mathrm{R}^{I_{2}} \ldots \otimes \mathrm{R}^{I_{N}}$ into a tensor subspace $\mathrm{R}^{P_{1}} \otimes \mathrm{R}^{P_{2}} \ldots \otimes \mathrm{R}^{P_{N}}$ with $\left(P_{n}<I n\right.$, for $\left.\mathrm{n}=1, \ldots, N\right)$.

We can define the projection of $n$-mode vector of $X_{m}$ as

$$
y_{m}=x_{m} \times \tilde{U}_{1}^{(n)^{T}} \times_{2} \tilde{U}^{(n)^{T}} \ldots \times_{N} \tilde{U}^{(N)^{\mathrm{T}}} .
$$

The tensor $y_{m}$ can capture most of the variations observed in the original tensor objects, assuming that these variations are measured by the total tensor scatter.

The objective of MPCA is the determination of the $N$ projection matrices $\mathrm{U}^{(\mathrm{n})}$ that maximize the total tensor scatter $\psi_{\mathrm{A}}$ as

$$
\left\{\tilde{\mathrm{U}}^{(\mathrm{n})}, n=1,2, \ldots, N\right\}=\underset{\tilde{\mathrm{U}}^{(1)}, \tilde{\mathrm{U}}^{(2)}, \ldots, \tilde{\mathrm{U}}^{(\mathrm{N})}}{\arg \max } \psi_{y}
$$

\section{COlor Face Hallucination with ERROR Regression MODEL IN MPCA}

In Fig. 2, we apply the MPCA to a training image set $\left\{x^{h}, x^{l}\right\}$ and training color face images can be defined as $x^{h} \in \mathrm{R}^{I_{1}} \otimes \mathrm{R}^{I_{2}} \ldots \otimes \mathrm{R}^{I_{N}}$ and $x^{l} \in \mathrm{R}^{J_{1}} \otimes \mathrm{R}^{J_{2}} \ldots \otimes \mathrm{R}^{J_{N}}$ which are the
HR color face image and LR color face image, respectively. Next, standard multi-linear algebra, any tensor can be defined as the product

$$
y^{h}=x^{h} \times_{1} \tilde{\mathrm{U}}^{h(1)^{\mathrm{T}}} \times_{2} \tilde{\mathrm{U}}^{h(2)^{\mathrm{T}}} \times_{3} \tilde{\mathrm{U}}^{h(3)^{\mathrm{T}}}
$$

And

$$
y^{l}=x^{l} \times_{1} \tilde{\mathrm{U}}^{l(1)^{\mathrm{T}}} \times{ }_{2} \tilde{\mathrm{U}}^{l(2)^{\mathrm{T}}} \times{ }_{3} \tilde{\mathrm{U}}^{l(3)^{\mathrm{T}}} .
$$

The tensor $y^{h}$ and $y^{l}$ can capture most of the variations observed in the original tensor objects, assuming that these variations are measured by the total scatter. Moreover, two sets of tensorPCA subspace projection are obtained, which are $y^{h}=\left[y_{r, s, t}^{h}\right]_{i}$ and $y^{l}=\left[y_{r, s, t}^{l}\right]_{i}$ respectively. We use $\left[y_{r, s, t}\right]$ to represent a tensor with $y_{r, s, t}$ as its $(r, s, t)$-th entry in RGB color space.

We can calculate the value of weight $w_{r, p}^{\prime}$ from the HR and LR of training sets. Each training image provides one equation to find $w_{r, p}^{\prime} \quad\left(p=1, \ldots, Q_{1} Q_{2} Q_{3}\right) \quad$ where $\left(Q_{1}<J_{1}, Q_{2}<J_{2}, Q_{3}<J_{3}\right)$. The Gaussian model is used to evaluate the weighted distance between the projection coefficients and defined as

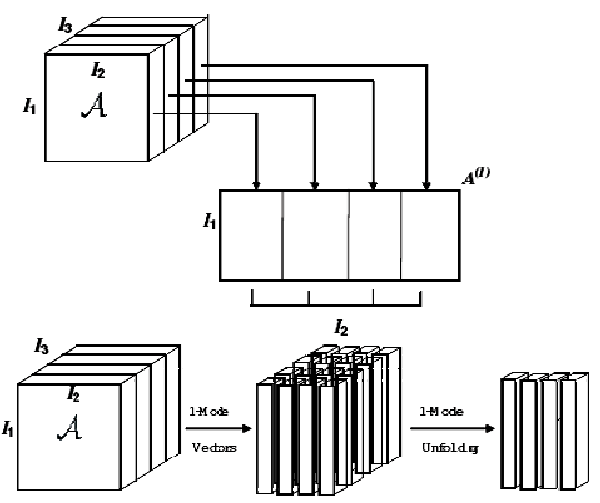

Fig. 1. Block diagram of proposed method

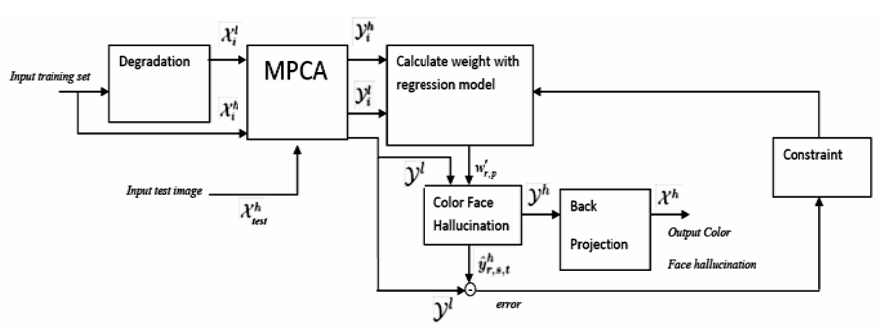

Fig. 2. Block diagram of proposed method

$$
P\left(y_{r}, y^{l}\right) \approx \operatorname{cexp}\left\{-\sum_{p=1}^{-0, Q_{2} Q_{3}} \frac{\left(y_{r}-w_{r, p} y_{p}^{l}\right)^{2}}{2}\right\}
$$

Next, we can apply Maximum Likelihood estimation to find $\hat{y}_{r}^{h}$ which can be expressed as

$$
\hat{y}_{r}^{h}=\underset{y_{r}}{\arg \max } P\left(y_{r} \mid y^{l}\right) .
$$

In (), we can rewrite in regression model as

$$
\hat{y}_{r}^{h}=\sum_{p=1}^{Q_{1} Q_{2} Q_{3}} w_{r, p}^{\prime} y_{p}^{l} .
$$


After the process of face hallucination, we can calculate the error between $\hat{y}_{r, s, t}^{h}$ and $y^{l}$ as

$$
\mathrm{e}=\hat{y}_{r, s, t}^{h}-y^{l}
$$

In our proposed technique, we use an error to be useful information. In error estimation, we can use the existing LR features in MPCA subspace or LR training dataset for regression analysis.

\section{EXPERIMENTAL RESULTS}

In this section, our face hallucination with MPCA in color facial image algorithm is tested against the tensorPCA which is applied in each color channel. We used face images from a subset of FERET databases to form two data sets for training and testing images in RGB color model.

The experiments are conducted with a large number of frontal face images from FERET data set [12], [13] and other collections, which consist of many different races, illuminations and types of face images. In our experiments, we randomly select 500 normal expression images of different persons on the same light condition and other 50 images are used for testing. According to demand, we manually crop the interesting region of the faces and unify the images to the size of $(30 \times 30)$. We show some example training faces from FERET database in Fig. 3.

In the degradation process, each testing image (LR) is introduced with Gaussian blur with variance 1 and resized by down-sampling 2:1 $(15 \times 15)$, then we add Gaussian noise with variance $10^{-6}$. To establish a standard training data set, we aligned these face images manually by hand, marking the location of 3 points: the centers of the eyeballs and the lower tip of the nose. These 3 points define an affine warp, which is used to warp the images into a canonical form.
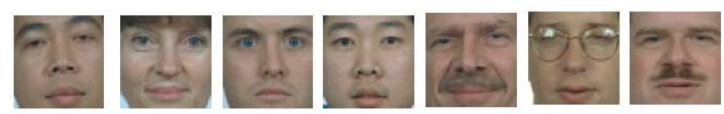

Fig. 3. Example training faces from FERET database in our proposed algorithm

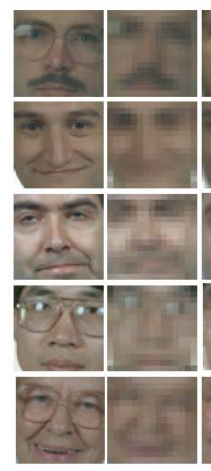

(a)

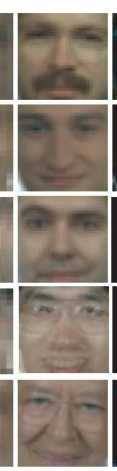

(b)

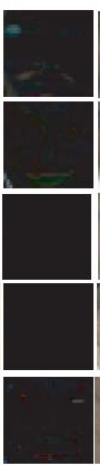

(d)

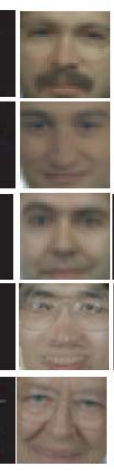

(e)

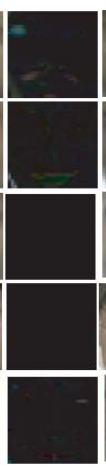

(f)

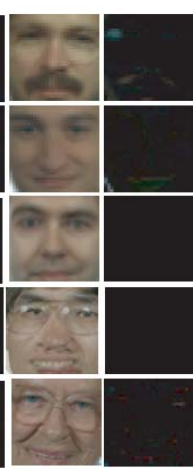

(g) (h)
Fig. 4. Some of experimental results (RGB color model) with an error regression model in MPCA method. (a) original HR images $(30 \times 30)$; (b) input LR images $(15 \times 15)$ with noise, motion and blur in LR images;(c) face hallucination result with 90 percent traditional PCA; (d) different image of face hallucination result with 90 percent traditional PCA; (e) face hallucination result with 90 percent tensorPCA; (f) different image of face hallucination result with 90 percent tensorPCA; $(\mathrm{g})$ face hallucination result with our proposed method; (h) different image of face hallucination result with our proposed method;

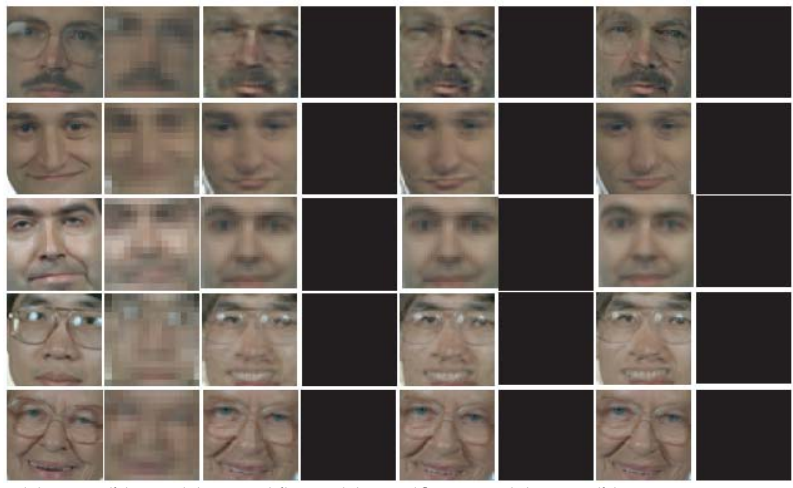
(a)
(b) (c)
(d) (e) (f)
(g) (h)

Fig. 5. Some of experimental results (RGB color model) with an error regression model in MPCA method. (a) original HR images $(30 \times 30)$; (b) input LR images $(15 \times 15)$ with noise, motion and blur in LR images; (c) face hallucination result with 95 percent traditional PCA; (d) different image of face hallucination result with 95 percent traditional PCA; (e) face hallucination result with 95 percent tensorPCA; (f) different image of face hallucination result with 95 percent tensorPCA; (g) face hallucination result with our proposed method; (h) different image of face hallucination result with our proposed method;

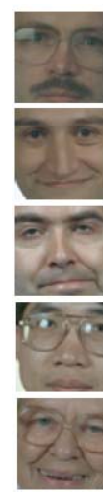

(a)

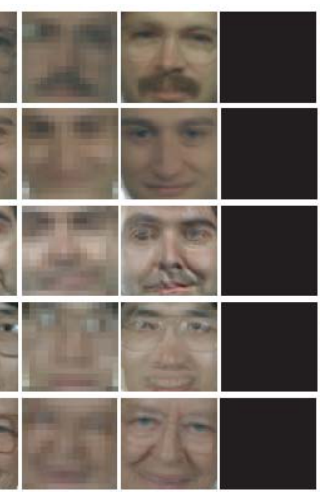

(b) (c)

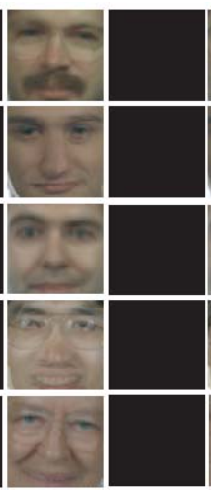

(e)

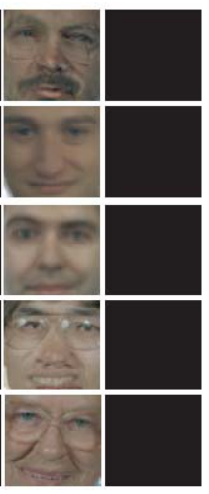

(g)

(h)
Fig. 6. Some of experimental results (RGB color model) with an error regression model in MPCA method. (a) original HR images $(30 \times 30)$; (b) input LR images $(15 \times 15)$ with noise, motion and blur in LR images; (c) face hallucination result with 100 percent traditional PCA; (d) different image of face hallucination result with 100 percent traditional PCA; (e) face hallucination result with 100 percent tensorPCA; (f) different image of face hallucination result with 100 percent tensorPCA; (g) face hallucination result with our proposed method; (h) different image of face hallucination result with our proposed method;

As shown in Fig. 4-6 (c) and (d), with traditional PCA method, we can observe that dirty disturbance in the global reconstructed images and the results have some noise around the eyes and mouth. In particular, if our proposed method is implemented with 100 percent of PCA, the results will become similar to the original HR facial images. Additionally, the details in our hallucination results such as eyes, noses, lips and eyebrows quite different from the original HR images.

\section{CONCLUSION}

In this paper, we propose a novel hallucination technique which can reconstruct the color face images in RGB color models. The color face images are presented in an error regression model and the multi-linear principal component analysis (MPCA) is applied to capture the most of the original tensorial input variation. For testing our proposed method, we used facial images from the FERET database to validate the algorithm. The experiments clearly 
analysis (MPCA) is applied to capture the most of the original tensorial input variation. For testing our proposed method, we used facial images from the FERET database to validate the algorithm. The experiments clearly demonstrated that we can generate photorealistic color face images by using the MPCA subspace with regression model and our hallucinated approach is suitable for RGB color spaces. We also implemented the hallucination method with tensorPCA in case of independent color channel in each color space such as RGB color model. The results showed that it can reconstruct the reasonable HR color face images. The performance of our novel color hallucination technique was observed to be better than that of the tensorPCA which is applied in each color channel.

\section{ACKNOWLEDGEMENT}

The authors are grateful to thank the Department of Electrical Engineering which is mainly supported by Faculty of Engineering Mahidol University. The research in this paper uses the FERET face Database collected under the sponsor by the Department of Defense's Counterdrug Technology Development Program through the Defense Advanced Research Products Agency (DARPA)

\section{REFERENCES}

[1] S. Baker and T. Kanade, "Hallucinating faces," IEEEInt. Conf. Automatic Face and Gesture Recog, pp. 83-88, 2000.

[2] K. N. Plataniotis and A. N. Venetsanopoulos, Color image processing and applications, Berlin, Germany: Springer-Verlag, 2000.

[3] K. W. Bowyer, K. Chang, and P. Flynn, "A survey Of approaches and challenges in $3 \mathrm{~d}$ and multi-modal $3 \mathrm{~d}+2 \mathrm{~d}$ face recognition," Comput. Vis. Image Understand, vol. 101, Jan. 2006.

[4] S. Z. Li, C. Zhao, M. Ao, and Z. Lei, "Learning to fuse $3 \mathrm{~d}+2 \mathrm{~d}$ based face recognition at both feature and decision levels," in Proc. IEEE Int.

[5] A. Colombo, C. Cusano, and R. Schettini, "3d face Detection using curvature analysis," Pattern Recognit, vol.39, no. 3, Mar. 2006.

[6] X. Tang and X. Wang, "Face sketch recognition," IEEE Trans. on Circuits, Systems, and Video Technology, vol. 14, no. 1, pp. 50-57, 2004.

[7] J. Wu and M. M. Trivedi, "A regression model in tensorpca subspace for face image super-resolution reconstruction," in Proc. IEEE Conf. Computer Vision and Pattern Recognition, vol.3, pp. 627-630, 2006.
[8] B. K. Gunturk, A. U. Batur, Y. Altunbasak, M. H. Hayes, and R. M. Mersereau, "Eigenface-domain super-resolution for face recognition," IEEE Trans. Image Process, vol.12, no. 5, pp. 597-606, May 2003.

[9] A. Chakrabarti, A. Rajagopalan, and R. Chellappa, "Super-resolution of face images using kernel pca-based prior," IEEE Trans. on Multimedia, vol. 9, pp. 888-892, 2007.

[10] C. Liu, "A Two-Step Approach to Hallucinating Faces:Global Parametric Model and Local Nonparametric Model," IEEE Trans. Image Process, 2001.

[11] H. Lu, K. N. K. Plataniotis, and A. N. Venetsanopoulos, "Multilinear principal component analysis of tensor objects," IEEE Transactions on Neural Networks, vol.19, no.1, pp.18-39, 2008.

[12] P. J. Phillips, H. Wechsler, J. Huang, and P. Rauss, "The FERET database and evaluation procedure for face recognition algorithms," Image and Vision Computing, vol.16, no. 5, 1998.

[13] P. J. Phillips, H. Moon, S. A. Rizvi, and P. J. Rauss, "The FERET evaluation methodology for face recognition algorithms," IEEE Trans. PAMI, vol. 22, 2000.

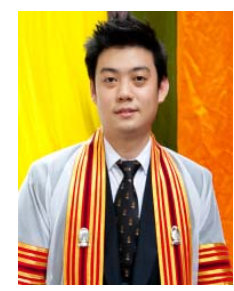

Krissada Asavaskulkiet received the B.Eng., M.Eng. and Ph.D. degrees in Electrical Engineering from the Chulalongkorn University, Thailand in 2001, 2004 and 20011 respectively. He is a lecturer in the Department of Electrical Engineering, Mahidol University, Thailand. His research areas are digital signal processing in image processing, face and automatic target recognition.

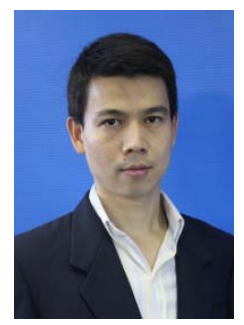

Phumin Kirawanich (S'02-M'04) received the M.S. and Ph.D. degrees in electrical engineering from the University of Missouri, Columbia, in 1999 and 2002,respectively. From 2002 to 2007, he was with the Power Electronics Research Center and the High Power Electromagnetic Radiation Laboratory, University of Missouri, as a Postdoctoral Fellow. From 2007 to 2008, he was with the Department of Electrical and Computer Engineering, University of Missouri, as a Research Assistant Professor. Since 2008, he has been a Faculty Member with the Department of Electrical Engineering, Mahidol University, Salaya, Thailand, as an Assistant Professor. He has contributed a number of research papers and articles to the book entitled Ultra-Wideband, Short-PulseElectromagnetics (Kluwer, 2007). His research interests include pulsed-power technology for biomedical and agricultural applications, terahertz-pulse generation, and electromagnetic and semiconductor device physics computation. 